\title{
Linear momentum and angular momentum transfer in the reactions of ${ }^{16} \mathrm{O}$ with ${ }^{154} \mathrm{Sm}$
}

\author{
M. N. Namboodiri* \\ Cyclotron Institute, Texas A\&M University, College Station, Texas 77843 \\ and Nuclear Chemistry Division, Lawrence Livermore National Laboratory, Livermore, California 94550 \\ R. K. Choudhury, ${ }^{\dagger}$ L. Adler, ${ }^{\ddagger}$ J. D. Bronson, D. Fabris, U. Garg, ${ }^{\S}$ P. L. Gonthier, ${ }^{* *}$ \\ K. Hagel, D. R. Haenni, Y. W. Lui, Z. Majka, G. Mouchaty, T. Murakami, ${ }^{\dagger \dagger}$ J. B. Natowitz, \\ G. Nebbia, R. P. Schmitt, S. Simon, J. P. Sullivan, and D. H. Youngblood \\ Cyclotron Institute, Texas A\&M University, College Station, Texas 77843 \\ (Received 13 December 1985; revised manuscript received 18 September 1986)
}

\begin{abstract}
From coincidence measurements between projectile-like fragments or heavy residues and their associated $\gamma$ rays, the angular momentum transfers for a variety of incomplete fusion reactions of 180 and $310 \mathrm{MeV}{ }^{16} \mathrm{O}$ with ${ }^{154} \mathrm{Sm}$ have been derived. At the higher energy, the correlation between angular momentum transfer and linear momentum transfer has been obtained over the entire range of linear momentum transfer. A comparison of the data with calculations of both the sum-rule and geometric overlap models indicates that each makes reasonable predictions of the observed trend even though the assumptions of the models are quite different, and very different initial partial waves are predicted to contribute to particular reaction channels. This results primarily from prescriptions relating fractional mass transfer to fractional angular momentum transfer. The reconstruction of the initial partial wave distributions from correlated measurements of linear momentum and angular momentum transfers is addressed. Comparisons are also made with more recent model calculations which focus on nucleon-nucleon scattering as the mechanism of momentum transfer.
\end{abstract}

\section{INTRODUCTION}

Measurements of angular momentum and linear momentum transfer in heavy ion collisions can provide a great deal of information on the systematic trends of reaction mechanisms in the intermediate energy region. ${ }^{1-6}$ One real utility of such mechanism studies is that they give one the ability to identify and study nuclei in reasonably well-defined conditions of excitation energy and angular momentum. Correlated measurements of linear momentum and angular momentum transfer offer the possibility of delineating the partial wave dependence of the dominant mechanisms. This allows one to make restrictive tests of various theoretical models of heavy ion collisions and also allows a much better characterization of the properties of the composite nuclei which are produced.

In order to explore the partial wave dependence of the dominant mechanisms, several techniques may be utilized. For example, the measurements of fission cross sections in concert with momentum transfer is one method which provides some information on the partial waves involved in incomplete fusion. Such an approach has been followed by Huizenga and co-workers. ${ }^{5}$ From an empirical decomposition of the folding angle distribution using information obtained from the out-of-plane, evaporationdominated, fragment correlations, they have derived cross sections for total momentum transfer (TMT) consistent with a dynamic model. Assuming these cross sections to represent only the lowest partial waves, they have further derived some information on the range of partial waves contributing to TMT and to incomplete fusion reactions.
Using both excitation function data and momentum transfer measurements at energies in the $20 \mathrm{MeV} /$ nucleon range, Gavron et al. $^{7}$ have also drawn some conclusions regarding the range of partial waves contributing to the dominant reactions. Again the arguments are based on cross section measurements alone.

While these results are interesting in their implications regarding the partial waves contributing to fusion and various incomplete fusion processes, they rely on cross section arguments and on the packing of cross section into well-defined minimum $l$ windows with little or no overlap. Certainly, some impact parameter localization for such reactions is predicted by several models, but the actual range of impact parameters contributing to a particular reaction may be quite different from that derived from cross section arguments alone.

Among the models which treat incomplete fusion, the sum-rule model of Wilczynski ${ }^{8,9}$ has been most widely applied. Recently, Harvey and co-workers have proposed an alternative geometric overlap model which makes some significant contrasting predictions to those of the sumrule model. ${ }^{10,11}$ Attempts to develop a more microscopic overlap model, based on nucleon-nucleon interactions, have been made by Harvey ${ }^{12}$ and Cole. ${ }^{13}$ Others, such as Udagawa and Tamura ${ }^{14,15}$ and Kerman and McVoy, ${ }^{16}$ have recently treated the question of massive transfer reactions in less phenomenological terms, but these latter models have not been widely applied to reactions of the type considered in this paper.

Several $\gamma$-ray multiplicity studies in the $6-10$ $\mathrm{MeV} /$ nucleon range ${ }^{4,9}$ have been interpreted as being in agreement with the Wilczynski model prediction of the 
average transfers. On the other hand, a comparison of the predictions of the Harvey model with $Q$ values, angular momentum transfers, and excitation functions for ejectile cross sections for the reactions of ${ }^{20} \mathrm{Ne}$ with ${ }^{197} \mathrm{Au}$ to energies as high as $20 \mathrm{MeV} /$ nucleon shows very good agreement, supporting the application of this model. ${ }^{10,11}$ In particular, this model successfully predicts the relative constancy of the cross sections at energies well above threshold, which is in contrast to the predicted decrease expected from the Wilczynski model. ${ }^{9}$ The Cole model also enjoys success in the prediction of the cross sections of projectile-like fragments.

Determining the range of applicability of each of these models in the intermediate energy region is obviously useful.

From measurements of (a) the average total $\gamma$-ray energy observed in coincidence with projectile-like fragments and (b) the average $\gamma$-ray multiplicity in coincidence with heavy residues, we have deduced angular momentum transfers in the reactions of 180 and $310 \mathrm{MeV}{ }^{16} \mathrm{O}$ with ${ }^{154} \mathrm{Sm}$. At $310 \mathrm{MeV}$ we made measurements using both these methods, whereas at $180 \mathrm{MeV}$ only the first type of measurement was done.

At $310 \mathrm{MeV}$ we have derived the correlation between linear momentum and angular momentum transfers over the entire range of linear momentum transfer. For the ejectile data at $310 \mathrm{MeV}$ it was necessary to apply significant corrections to account for the missing momentum carried away by undetected particles since the observed ejectile is not necessarily the primary ejectile. This problem did not occur in the simultaneous measurements of the residue momentum and the $\gamma$-ray multiplicity. In such cases, the transfer of both linear and angular momentum to the heavy product is determined. The two measurements taken together offer the possibility of reconstruction of the initial partial wave dependence, given certain simple assumptions regarding the relationship between linear momentum and angular momentum transfer.

\section{EXPERIMENTAL DETAILS}

The experiments were performed using ${ }^{16} \mathrm{O}$ beams from the Variable Energy Cyclotron at Texas A\&M University. The experimental arrangements are shown in Figs. 1 and 2.

\section{A. Measurements of ejectile-gamma ray coincidences}

A self-supporting ${ }^{154} \mathrm{Sm}$ target $1.0 \mathrm{mg} / \mathrm{cm}^{2}$ thick was employed. The target location was at the center of a 16segment $\mathrm{NaI}(\mathrm{Tl})$ total energy crystal (TEC) array shown in Fig. 1. Measurements were performed at bombarding energies of 180 and $310 \mathrm{MeV}$. Fragments with atomic numbers from 2 to 10 were detected at a laboratory angle of $12^{\circ}$ with respect to the beam direction using two telescopes consisting of 50,100, and $1000 \mu \mathrm{m}$ Si detectors.

Either particle detector telescope was used as the trigger for accepting events to be recorded. Energy signals from the silicon detectors and from the individual $\mathrm{NaI}$ segments were recorded for each event. In addition, the time difference between the detection of a particle in either sil-
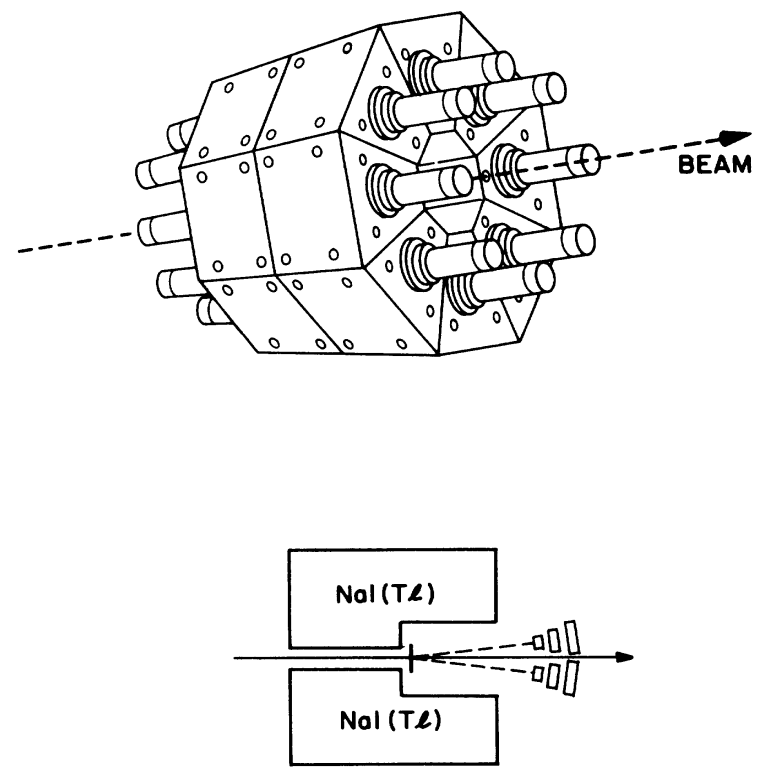

FIG. 1. Schematic diagram of the total energy crystal measurements. At top is an artist's view of the total energy crystal. At bottom is a schematic showing the position of the target and the particle telescopes.

icon telescope and a gamma ray in each of the NaI segments was also digitized and recorded for every event. Monitoring the individual time spectra enabled us to ensure that pile-up effects were low during the experiment. Data acquisition was performed using CAMAC instrumentation and a VAX-11/780 computer. The data were recorded event by event on magnetic tape for off-line analysis. Isotopic identification of the ejectiles was performed using the algorithm given by Butler et al. ${ }^{17}$ This method gave isotope resolution for all products of $Z \leq 8$ that penetrated to the third detector in the telescopes.

In the compact configuration used in the present experiment, the TEC has a geometrical solid angle of approximately $90 \%$. Energy calibration and the response characteristics of each segment in the TEC assembly were determined using gamma ray sources of energy up to $1.9 \mathrm{MeV}$

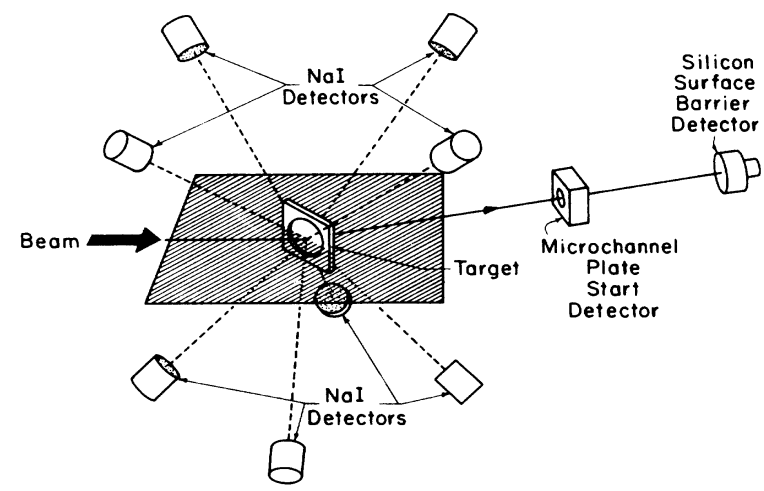

FIG. 2. Schematic diagram of measurements using the multiplicity filter and time-of-flight arm. 
and the 4.4 MeV gamma ray from the inelastic scattering of ${ }^{4} \mathrm{He}$ from a carbon target. We should emphasize that the vicinity of $1 \mathrm{MeV}$ is the most important energy region in this connection, since the average energy of the gamma rays emitted by the heavy residues in the reactions considered here is about $1 \mathrm{MeV} .^{18}$ The overall detection efficiency and the photopeak efficiency for the TEC assembly were $83.5 \%$ and $62.7 \%$, respectively, for $662 \mathrm{keV}$ gamma rays and $74.5 \%$ and $40.4 \%$, respectively, for $1332 \mathrm{keV}$ gamma rays. In addition to several calibration experiments in the actual geometry used, detailed simulations of the response of the crystal assembly have also been made in our laboratory. ${ }^{19}$

It is worth emphasizing that the TEC is segmented and is not run as a simple sum spectrometer. During the off line analysis, the total gamma energy in each event was determined by summing the energy deposited in each of the segments. The spectrum of gamma rays emitted in coincidence with each ejectile isotope was determined for $10 \mathrm{MeV}$ intervals in the ejectile energy. Using the time spectrum for each $\mathrm{NaI}$ segment, corrections were made for random coincidences. The compact geometry of the TEC did not allow neutron-gamma discrimination by time-of-flight methods. No special correction was made for neutrons detected by the TEC. However, we conclude that the contributions from neutrons to the total gamma energy is small (less than $\sim 5 \%$ ) from the very good agreement between the angular momentum transfer derived from our total gamma energy measurements and that obtained from gamma multiplicity measurements for the very similar system ${ }^{16} \mathrm{O}+{ }^{150} \mathrm{Sm}$ at $310 \mathrm{MeV}$ by Schmitt et al., ${ }^{20}$ in which corrections were made for neutrons (see Fig. 7 below).

\section{B. Residue- $\gamma$ coincidence experiments}

In this case a $250 \mu \mathrm{g} / \mathrm{cm}^{2}{ }^{154} \mathrm{Sm}$ self-supporting foil was bombarded with beams of $310 \mathrm{MeV}{ }^{16} \mathrm{O}$. A microchannel plate (MCP) - silicon detector time-of-flight (TOF) system with a $50 \mathrm{~cm}$ flight path was employed to measure the residue velocities and energies. A gamma ray multiplicity filter consisting of eight $7.6 \times 7.6 \mathrm{~cm}^{2} \mathrm{NaI}$ counters oriented $45^{\circ}$ out of plane was used in coincidence with the TOF arm to measure gamma ray multiplicities of the residues. The experimental arrangement is shown in Fig. 2.

Data were written event by event on magnetic tape for later analysis off line with the computer code LISA.

Figure 3 represents a typical raw uncalibrated energy versus TOF spectrum. The wide arc in the lower region contains the residue events, and events in the top part correspond to projectile-like fragments. The higher velocity events actually come at larger times in this setup because we chose to start the time with the silicon detector. The flight path of $50 \mathrm{~cm}$ gave a most probable recoil time of flight of about $110 \mathrm{~ns}$ for our system. The time resolution in the MCP-Si system was about $700 \mathrm{ps}$; this rather poor resolution was mainly due to the silicon detector used.

To correct for the average plasma delay associated with low energy heavy residues in the TOF silicon detector, we have performed a separate experiment with a microchan-

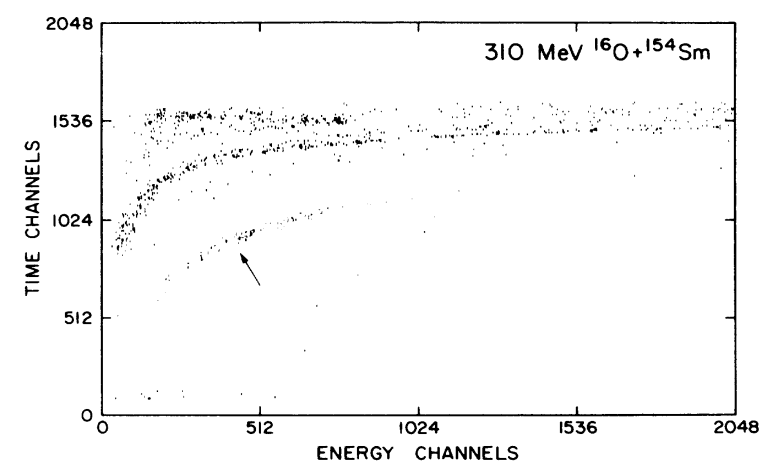

FIG. 3. Time-of-flight vs energy for products detected with the time-of-flight arm. The curved band of products corresponding to heavy residues is indicated by an arrow.

nel plate and parallel plate avalanche counter (PPAC) TOF system where the problem of plasma delay is not present. The $t_{0}$ for this experiment was obtained by performing the same velocity measurement with two different lengths of the TOF arm. The measurement was checked by performing a timing calibration using fission fragments and alpha particles from a ${ }^{252} \mathrm{Cf}$ source. We have found that the recoil residue TOF spectra from the MCP-PPAC and MCP-Si experiments overlapped each other essentially completely by a shift of 1.5-2.0 ns along the time axis, indicating a constant plasma delay correction with little dependence on the mass and energy of the heavy residue. This correction is indeed small compared to the most probable recoil TOF of $110 \mathrm{~ns}$. The measured recoil velocities were also corrected for the energy loss in the target assuming average values for the mass and atomic number of the recoils estimated from statistical model calculations of the deexcitation of the primary products. This correction amounted to less than $2 \%$.

An accurate determination of the masses of the recoils was not attempted in this experiment in view of the difficulty of measuring the low kinetic energies of the recoils.

The total efficiency of the multiplicity filter was about $10 \%$. Full details about the setup and the analysis of multiplicity data are given in Ref. 21. A correction was made for the contribution to the gamma multiplicity from the detection of neutrons. This correction was given by $\bar{n} \bar{\Omega}_{\mathrm{n}} / \bar{\Omega}_{\gamma}$, where $\bar{n}$ is the average neutron multiplicity (estimated from the excitation energy of the emitting system) and $\bar{\Omega}_{n} / \bar{\Omega}_{\gamma}$ the average efficiency ratio for neutrons and gamma rays, for which a value of 0.1 was used. We did not make any corrections for the gamma ray angular distributions as the alignment in the various incomplete fusion reactions leading to the residues is, in general, unknown. The corrections are, however, expected to be small.

\section{EXPERIMENTAL RESULTS}

\section{A. Ejectile $\left\langle E_{\gamma}\right\rangle$ measurements}

Energy spectra for various isotopes observed at $12^{\circ}$ are shown in Figs. 4 and 5. It is seen that at this forward an- 
gle the spectra of isotopes close to the projectile in $Z$ and $A$ peak at energies slightly less than those corresponding to the projectile velocity. For lighter ejectiles, the spectra consist of broad peaks covering a wide range of energy loss. The thresholds in the spectra represent the particle energies required to penetrate the first two detectors (150 $\mu \mathrm{m} \mathrm{Si}$ ) in the telescope.
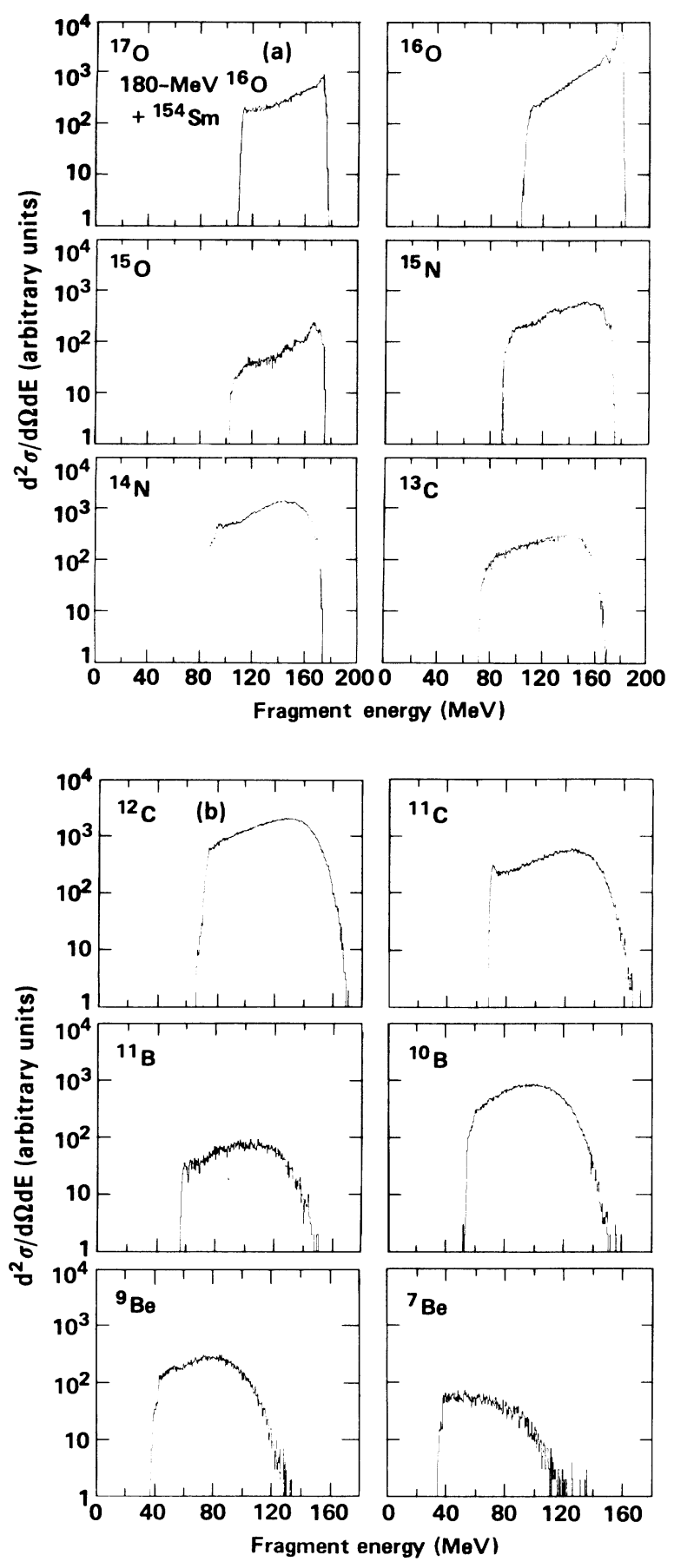

FIG. 4. Isotopic energy spectra for projectile-like fragments in the reactions of $180 \mathrm{MeV}{ }^{16} \mathrm{O}$ with ${ }^{154} \mathrm{Sm}$. The detector telescope was at $\theta_{\mathrm{lab}}=12^{\circ}$.
The average total gamma energies $\left\langle E_{\gamma}\right\rangle$, corresponding to $10 \mathrm{MeV}$ bins in the ejectile energy, are plotted in Fig. 6 as a function of the laboratory energy of the ejectile for selected isotopes. We estimate that the overall uncertainties in these $\left\langle E_{\gamma}\right\rangle$ values are less than $10 \%$. The general
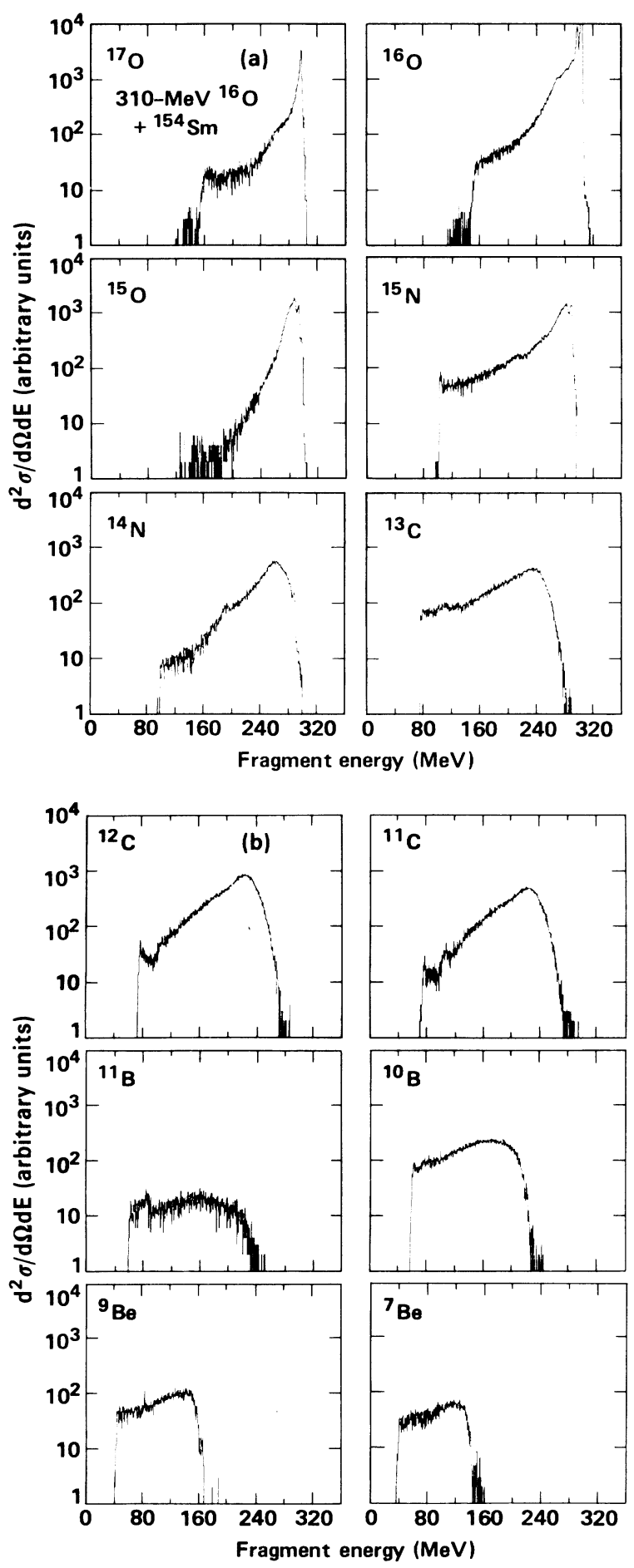

FIG. 5. Isotopic energy spectra for projectile-like fragments in the reactions of $310 \mathrm{MeV}{ }^{16} \mathrm{O}$ with ${ }^{154} \mathrm{Sm}$. The detector telescope was at $\theta_{\mathrm{lab}}=12^{\circ}$. 
trend in Fig. 6 is that of increasing $\left\langle E_{\gamma}\right\rangle$ with increasing energy loss. Similar upper limits for the gamma ray energies are reached at both projectile energies.

The $\gamma$-ray energy spectra from the total energy crystal indicated generally small to negligible contributions that could be identified as arising from deexcitation of projectile-like fragments. This is consistent with the small number of bound excited levels in many of the projectile fragments and also the experimental observation of Morissey et al. ${ }^{22}$ in the system ${ }^{14} \mathrm{~N}+\mathrm{Ag}$ at 35 $\mathrm{MeV} /$ nucleon that the fractions of the $\mathrm{Li}$ and $\mathrm{Be}$ fragments in bound excited states were small. In view of this, we have taken the observed average $\gamma$-ray energy as

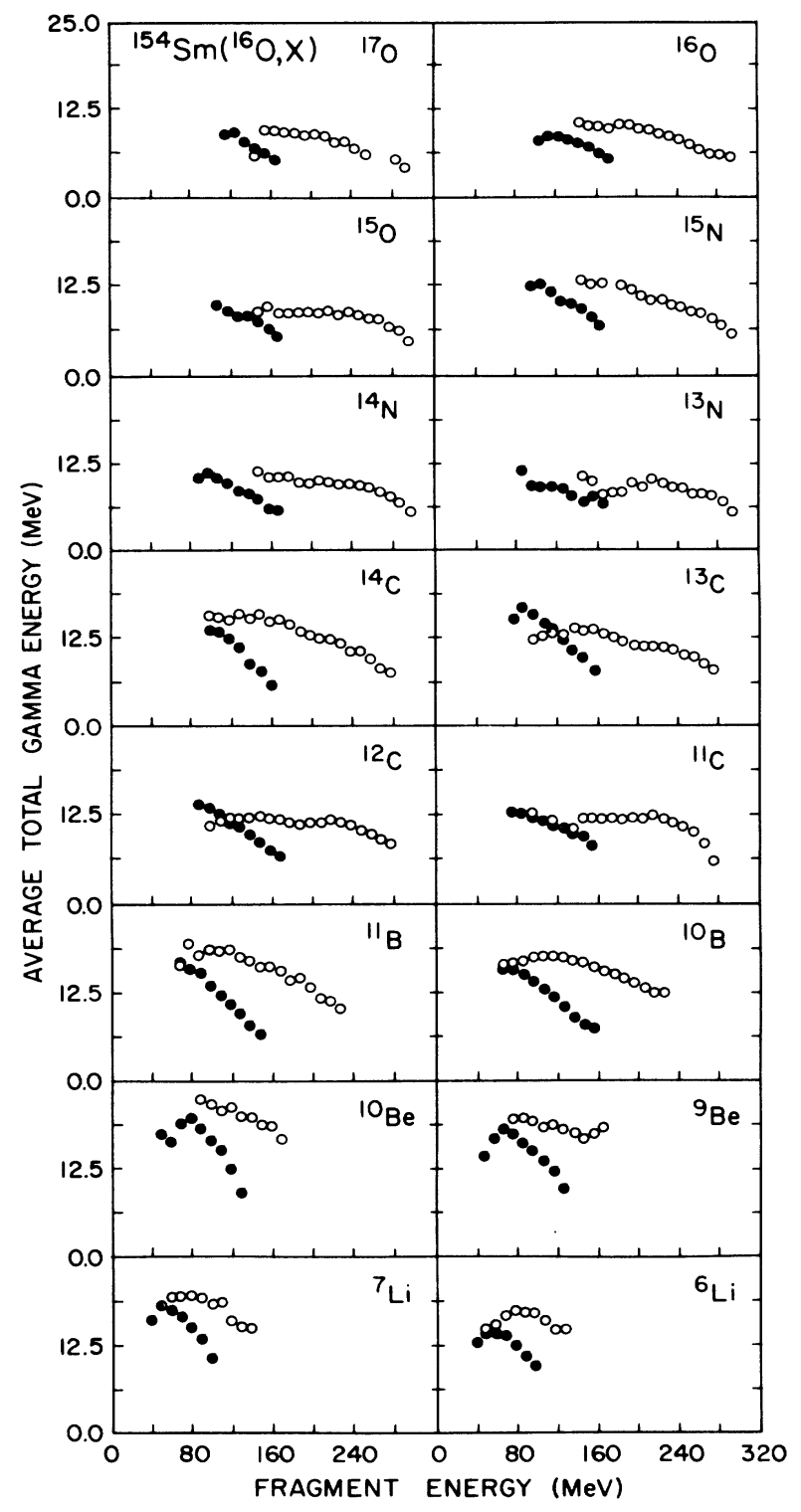

FIG. 6. Average total gamma-ray energy as a function of fragment energy for projectile-like fragments observed at $\theta_{\text {lab }}=12^{\circ}$. The averages were determined for $10 \mathrm{MeV}$ increments in the laboratory fragment energy. Solid circles, $180 \mathrm{MeV}$; open circles, $310 \mathrm{MeV}$. representative of the deexcitation of the target-like fragment. From these average $\gamma$-ray energies, values were then derived for the average angular momentum transfer as a function of observed ejectile energy.

For this transformation from average total gamma energies to angular momenta, we used the systematics of the average multiplicities and the average energies of gamma rays from fusion reactions. Details of this method are given in Ref. 18. Although the utility of $\gamma$ rays to probe the angular momenta of the residues may diminish at higher energies, the reactions studied here are primarily incomplete fusion reactions leading to excitation energies and angular momenta in the ranges for which the systematics have been established.

The values of the angular momentum transfer determined in this manner are displayed in Fig. 7 for isotopes with $Z>2$. We estimate the overall uncertainty in these angular momentum values to be about $15 \%$. In Fig. 7 we also show the results for several ejectiles in the very similar system $310 \mathrm{MeV}{ }^{16} \mathrm{O}+{ }^{150} \mathrm{Sm}$ obtained by Schmitt

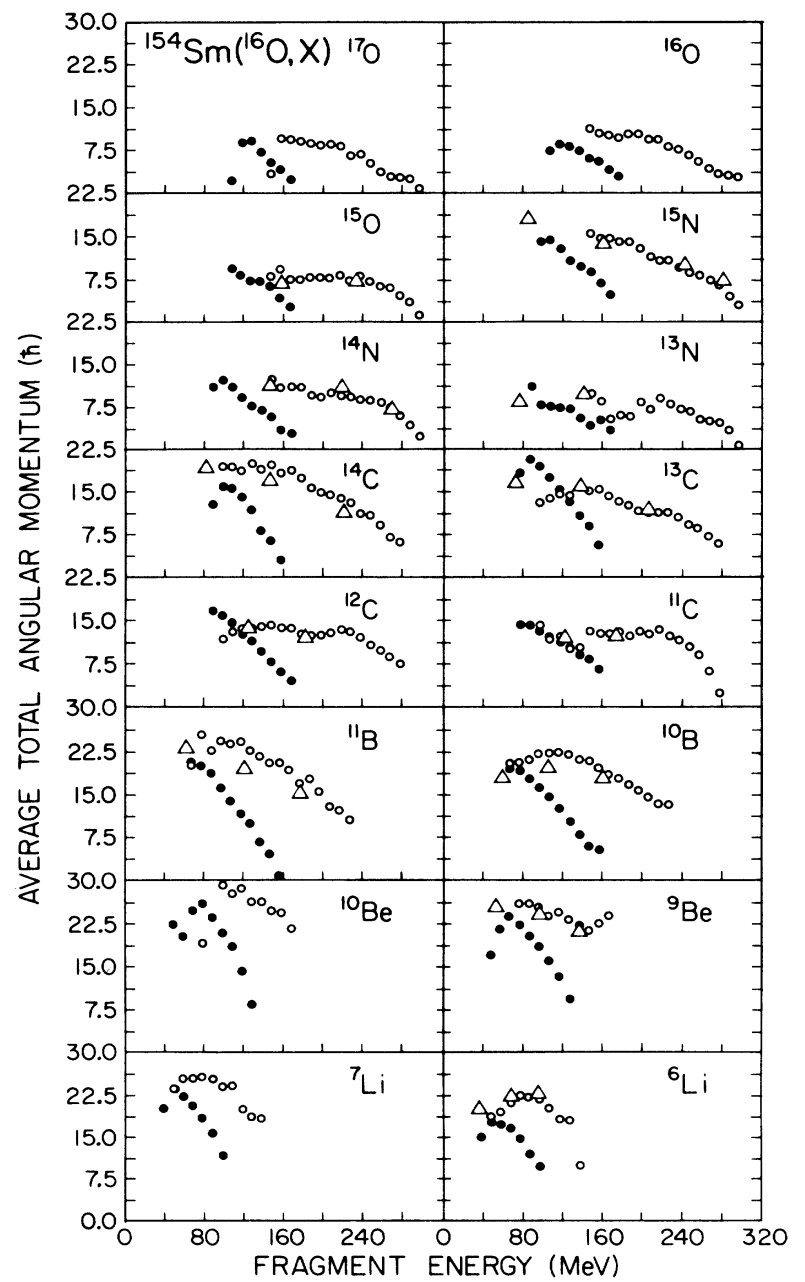

FIG. 7. Average total angular momentum as a function of fragment energy for projectile-like fragments observed at $\theta_{\text {lab }}=12^{\circ}$. Solid circles, $180 \mathrm{MeV}$; open circles, $310 \mathrm{MeV}$; open triangles, results from Ref. 20. 
et al. ${ }^{20}$ using gamma multiplicity measurements. Their multiplicity data correspond to ejectile energy bins of 5 $\mathrm{MeV} /$ nucleon width. As already mentioned above, these multiplicity measurements were corrected for neutrons. The agreement between the results of Schmitt et al. and our results for ${ }^{16} \mathrm{O}+{ }^{154} \mathrm{Sm}$ is very good, in general. This lends support to the use of the total gamma energy as an index of angular momentum transfer. In a separate measurement using the TEC, we found that the average angular momentum associated with $\alpha$-particle emission at both bombarding energies was $21 \hbar$ at the peak of the alpha spectrum. This measurement was done in exactly the same manner as discussed above, except that a threeelement telescope consisting of a gas ionization $\Delta E$ counter, a $1 \mathrm{~mm}$ silicon detector, and a $5 \mathrm{~mm} \mathrm{NaI}$ backdetector was used, enabling us to obtain more complete energy spectra than with the silicon detector telescopes.

The transferred angular momenta in Fig. 7 are seen to increase with decreasing ejectile mass, as would be expected, in general, for increasing mass transfer to the target. For individual ejectiles the transferred angular momentum is seen to increase and then apparently to saturate, with decreasing ejectile energy.

In Fig. 8 we show $l$-transfer distributions obtained in

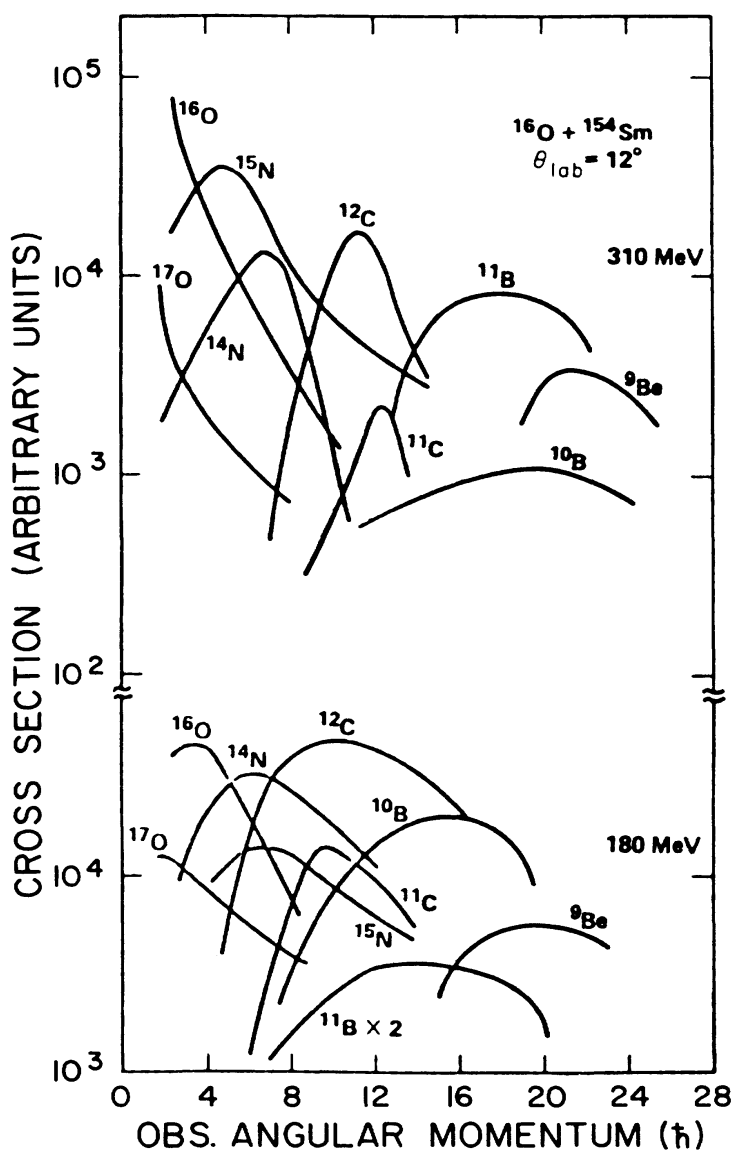

FIG. 8. Distribution of transferred angular momentum for projectile-like fragments observed at $\theta_{\mathrm{lab}}=12^{\circ}$. At top are the results from the irradiations with $310 \mathrm{MeV}{ }^{16} \mathrm{O}$. At the bottom are the results from the irradiations with $180 \mathrm{MeV}{ }^{16} \mathrm{O}$. the TEC experiments for several exit channels at both 180 and $310 \mathrm{MeV}$ for events leading to ejectile emission at $\theta_{\text {lab }}=12^{\circ}$. The curves drawn in these figures represent the smooth trends shown by the number of ejectile- $E_{\gamma}$ coincidences for each $10 \mathrm{MeV}$ bin in ejectile energy when plotted against the average $l$ transfer for that bin. Isotopes of the lightest $Z$ 's, for which the measured energy spectra did not cover a wide enough energy range, are not represented in the figures.

\section{B. Residue $\left\langle\boldsymbol{M}_{\gamma}\right\rangle$ measurements}

Figure 9 shows both the residue velocity distribution and $\left\langle M_{\gamma}\right\rangle$ as a function of velocity for the reactions of $310 \mathrm{MeV}{ }^{16} \mathrm{O}$ with ${ }^{154} \mathrm{Sm}$. The asymmetric tail at lower residue velocities represents the incomplete fusion reactions. Even the most probable momentum transfer, probably resulting from central collisions, is significantly less than full momentum transfer, as is noted by comparison

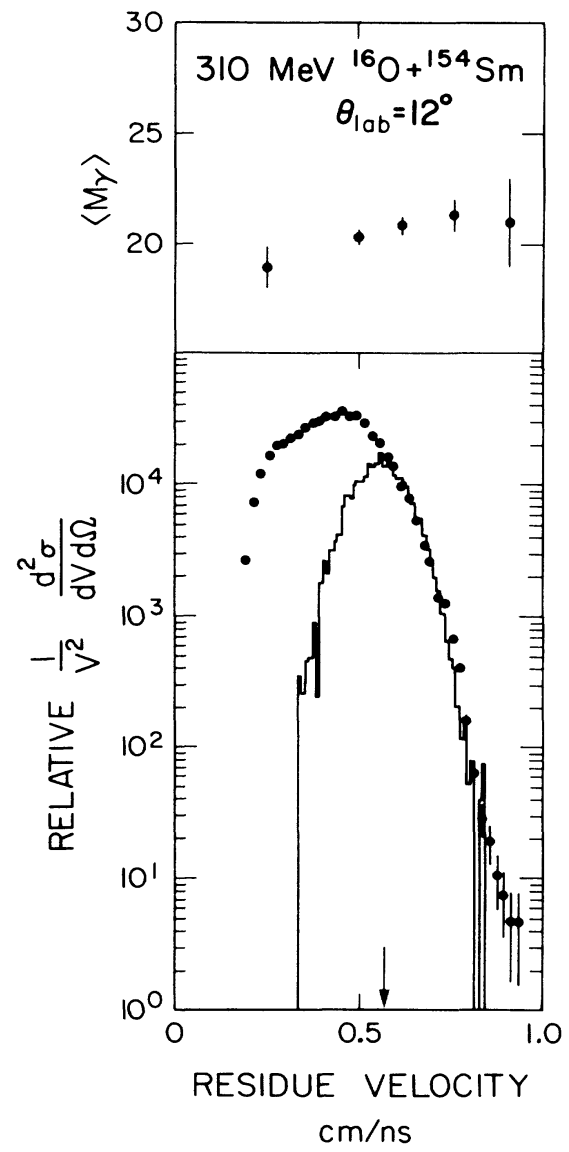

FIG. 9. Residue velocity distributions and average multiplicities for the reactions of $310 \mathrm{MeV}{ }^{16} \mathrm{O}$ with ${ }^{154} \mathrm{Sm}$. At the bottom of the figure is the experimentally determined residue velocity distribution at $\theta_{\mathrm{lab}}=12^{\circ}$ (solid circles). The histogram represents statistical model calculations of the residue distribution corresponding to complete fusion. The arrow indicates the velocity corresponding to total momentum transfer. At the top of the figure average gamma-ray multiplicities for selected residue velocities are presented. 
with the results of a calculation assuming total momentum transfer and statistical decay of the composite system. This calculation, for residues observed at $12^{\circ}$, was performed using the code LILITA (Ref. 23) and is presented in Fig. 9. The calculation has been arbitrarily normalized to the experimental data and provides an indication of both the possible fraction of complete fusion and the broadening of the primary momentum distribution which results from evaporative deexcitation. The variation of the gamma multiplicity with momentum transfer implied by Fig. 9 is somewhat flatter than expected from results such as those of Wilczynski et al. ${ }^{9}$ for the system ${ }^{14} \mathrm{~N}+{ }^{159} \mathrm{~Tb}$ at $140 \mathrm{MeV}$. Part of the reason for this flatness is the evaporative broadening of the velocity distribution which causes higher LMT (and angular momentum transfer) events to be assigned to lower apparent LMT (linear momentum transfer). Such a smearing of different incomplete fusion processes does not occur in the method of Ref. 9, where discrete gamma rays of the residues were used for defining the reaction channels.

\section{MODEL COMPARISONS}

The Wilczynski sum-rule model ${ }^{8,9}$ attributes incomplete fusion reactions to "hard grazing" collisions in which transfer of mass can occur only if the angular momentum of relative motion of the captured fragment with respect to the absorbing nucleus is smaller than the critical angular momentum of that fragment. It is assumed that the target-projectile system reaches quasiequilibrium and that the relative probabilities of different mass transfers allowed by the angular momentum criterion are governed by phase-space considerations. The captured fragment carries with it a fraction of the total angular momentum equal to its fraction of the projectile mass. The critical angular momenta calculated correspond roughly to the condition of vanishing pockets in the nuclear + Coulomb + centrifugal potential. In this model there is no frictional force which would allow higher partial waves to be damped and contribute to complete fusion.

In Fig. 10(a) are shown some representative $l$-wave distributions calculated for several primary ejectile channels by the Wilczynski model. The parameters employed are the same as those of Ref. 9.

The model of Harvey and co-workers ${ }^{10,11}$ is a geometric overlap model in which the portion of the projectile nucleus which is captured is taken to be the spherical cap sheared from the projectile by the target as it follows its interaction trajectory. This captured mass is assumed to carry a fraction of the angular momentum, which is defined by the relative angular momentum of the center of mass of the spherical cap. Thus the fraction of the orbital angular momentum which is captured is less than the mass ratio and varies with the relative magnitude of $r_{t}$, the effective radius for transfer, and $r_{e}$ the effective radius for the uncaptured primary ejectile.

Figure 10(b) depicts representative $l$-wave distributions, calculated using the overlap model as described in Ref. 11, for the same ejectiles as in Fig. 10(a). One immediately notes that, in general, the partial waves predicted to lead to a particular ejectile are much larger in this model than in the sum-rule model.

More recently, Harvey ${ }^{12}$ has proposed a microscopic model of incomplete fusion based on nucleon-nucleon collisions. In this model nucleons are removed from the projectile through nucleon-nucleon scattering. The effective cross sections for such scattering and the diffusivities of the nucleon distributions are important parameters in this calculation. A Monte Carlo approach is employed to determine the mass removed from the target. In an approach also based on nucleon-nucleon scattering, Cole ${ }^{13}$ has derived analytical formulas for the fragment yields, energy spectra, and angular distributions in incomplete fusion. Both authors have used these nucleon-nucleon scattering models to address the question of angular momentum transfer. ${ }^{13,24}$

Figure 10(b) shows the most probable initial $l$ values for various ejectiles predicted by Harvey's microscopic model with a nucleon mean free path of $13 \mathrm{fm}$. These calculations predict $l$ values similar to those of the geometric overlap model. The most probable initial $l$ values given by the model of Cole, also shown in Fig. 10(b), are found

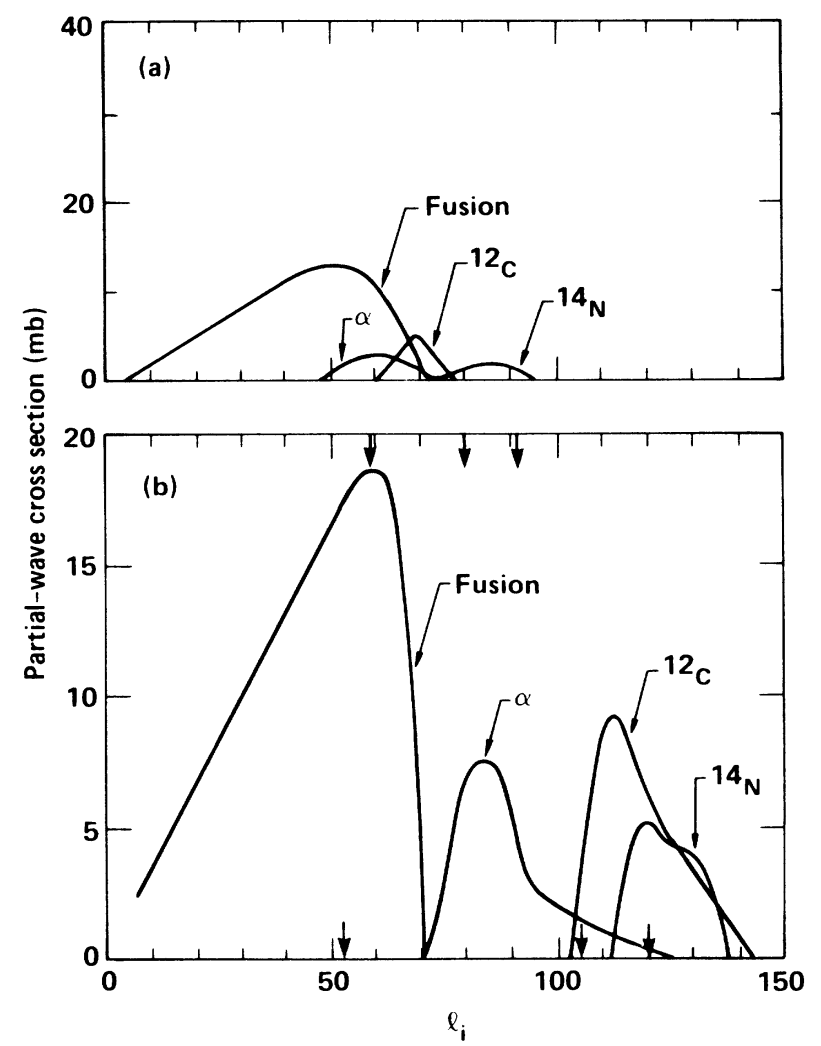

FIG. 10. (a) Wilczynski sum-rule predictions of the initial partial wave distributions leading to selected reaction channels. (b) Harvey-Homeyer geometric overlap model predictions of the initial partial wave distributions leading to selected reaction channels. The downward pointing arrows along the bottom $X$ axis represent the most probable initial $l$ values predicted by Harvey's microscopic model with a nuclear mean free path of 13 fm and those along the top $X$ axis the most probable $l$ values predicted by Cole's model. These arrows correspond, from left to right, to the ejectiles ${ }^{4} \mathrm{He},{ }^{12} \mathrm{C}$, and ${ }^{14} \mathrm{~N}$, respectively. 
to be generally lower than those predicted by Harvey.

One representation of the results of the $\left\langle E_{\gamma}\right\rangle$ measurements may be presented as derived angular momentum as a function of ejectile mass. This is done in Figs. 11 and 12 for the two bombarding energies. The data correspond to the average angular momentum transfer associated with the most probable ejectile energy. The data are represented as solid circles. For comparison we present the predictions of the Wilczynski sum-rule model and the Harvey-Murphy ${ }^{10}$ overlap model. It will be noted that these models lead to similar predictions of angular momentum transfer even though the initial partial waves involved are quite different. (It is worth pointing out that the overlap model given in Refs. 10 and 11 differ by about $20 \%$ in the initial $l$ values leading to the same ejectiles due to the differences in the evaluation of the Coulomb potential, although the distance at which the transfer takes place is calculated to be the same in both versions. Reference 10 addresses the question of angular momentum transfer explicitly, while Ref. 11 deals mainly with ejectile cross sections.) It will be noted further that the disagreement between the model predictions and experimental results, as presented, is significantly greater at 20 $\mathrm{MeV} /$ nucleon. This is not surprising since many previous experiments have established the importance of fast particle emission in collisions induced by $20 \mathrm{MeV} /$ nucleon heavy on projectiles. ${ }^{25-28}$ These particles may originate either from the breakup of primary ejectiles or in preequilibrium emission from the composite system. The existence of significant probabilities for the first process,

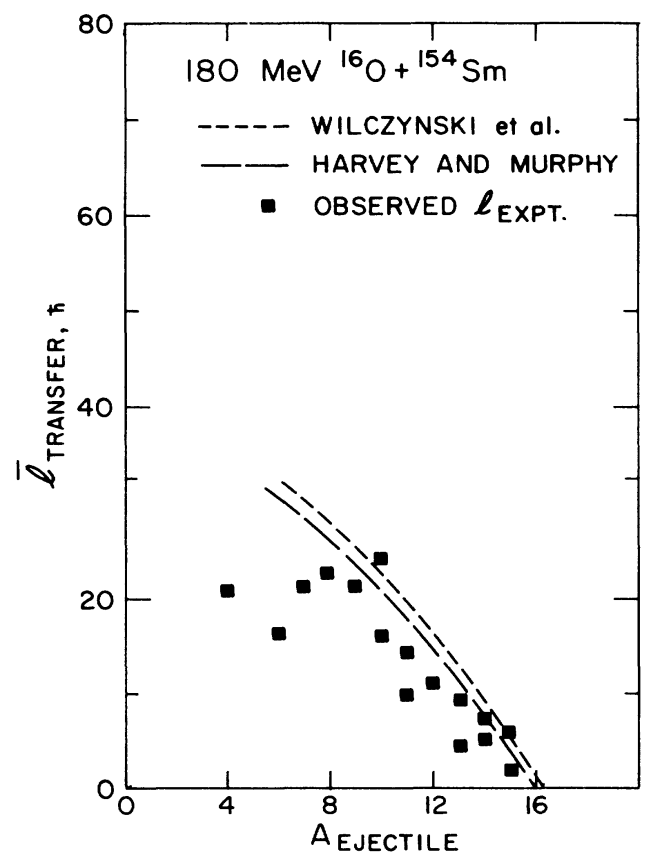

FIG. 11. Average transferred angular momentum as a function of the mass of the observed ejectile for the reactions of 180 $\mathrm{MeV}{ }^{16} \mathrm{O}$ with ${ }^{154} \mathrm{Sm}$. The experimental results are represented by solid squares. Predictions of the sum-rule and geometric overlap models assuming the observation of primary ejectiles are also presented. ejectile breakup, complicates the interpretation of incomplete momentum transfer experiments in which the ejectiles are studied since they are often not the primary ejectiles but rather residues resulting from the breakup.

The importance of such processes in the reactions of $310 \mathrm{MeV}{ }^{16} \mathrm{O}$ with ${ }^{238} \mathrm{U}$ has been clearly demonstrated by Back et al. ${ }^{28}$ in coincidence experiments in which the momentum transfer in incomplete fusion reactions was determined from measurement of folding angles of fission fragments observed in coincidence with the projectile-like fragments.

Written in terms of $P_{i}$, the original projectile momentum, $P_{3}^{\|}$, the component of the observed ejectile momentum parallel to the beam axis and $P_{r}^{\|}$, the parallel momentum component of the heavy partner as determined from the folding angle measurements, it is possible to express the average fractional momentum transfer as

$$
\frac{\left\langle P_{r}^{\|}\right\rangle}{P_{i}}=0.03+\frac{2}{3}\left(1-\frac{\left\langle P_{3}^{\|}\right\rangle}{P_{i}}\right) .
$$

In the following analysis of the ejectile data for the reactions of $310 \mathrm{MeV}{ }^{16} \mathrm{O}$ with ${ }^{154} \mathrm{Sm}$, we have assumed that the fractional momentum transfer follows exactly the same relationship as that observed by Back et al. for 310 $\mathrm{MeV}{ }^{16} \mathrm{O}+{ }^{238} \mathrm{U}$. The similarity of fragment energy spectra and relative isotopic yields for ${ }^{16} \mathrm{O}$ induced reactions on different targets in this mass range supports this approximation. ${ }^{28,29}$

It has already been noted that the energy spectra in Figs. 3 and 4 exhibit peaks. In order to explore the dependence of the average angular momentum transfer on

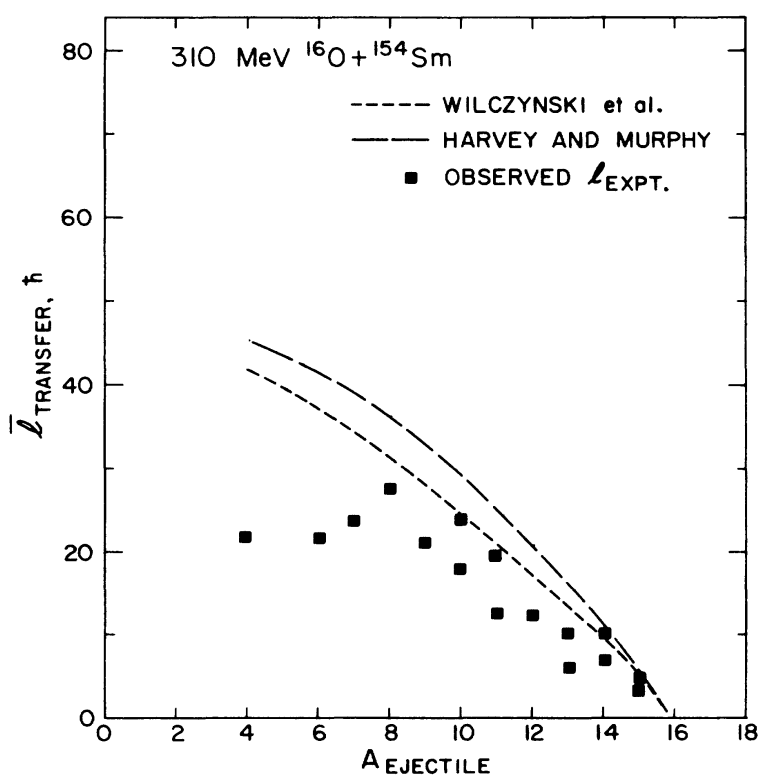

FIG. 12. Average transferred angular momentum as a function of the mass of the observed ejectile for the reactions of 310 $\mathrm{MeV}{ }^{16} \mathrm{O}$ with ${ }^{154} \mathrm{Sm}$. The experimental results are represented by solid squares. Predictions of the sum-rule and geometric overlap models assuming the observation of primary ejectiles are also presented. 
linear momentum transfer, we focus on the measurements of $\left\langle E_{\gamma}\right\rangle$ obtained in coincidence with the ejectiles of most probable energy, taking them to be characteristic of the reaction. We then determine the actual fractional momentum transfer associated with observed ejectiles of the most probable energy by using Eq. (1). Thus we correct for the missing momentum.

The resulting correlation between the average angular momentum transfer and the average fractional linear momentum transfer, derived in the manner described, is plotted in Fig. 13. Solid circles are used to represent these results.

As we have seen, an alternative for correlating linear momentum and angular momentum transfer is by observation of the heavy residue velocities in coincidence with the $\gamma$-ray multiplicities. The solid squares in Fig. 13 represent the results of our residue- $\left\langle M_{\gamma}\right\rangle$ measurements for this system. In this case we have derived the fractional momentum from the residue velocity using the relation$\operatorname{ship}^{30}$

$$
P=\frac{A_{t}}{A_{p}}\left(\frac{V_{R}}{V_{p}-V_{R}}\right),
$$

where $A_{t}$ and $A_{p}$ are the target and projectile masses, $V_{R}$ and $V_{p}$ are the residue and projectile velocities, and $P$ is

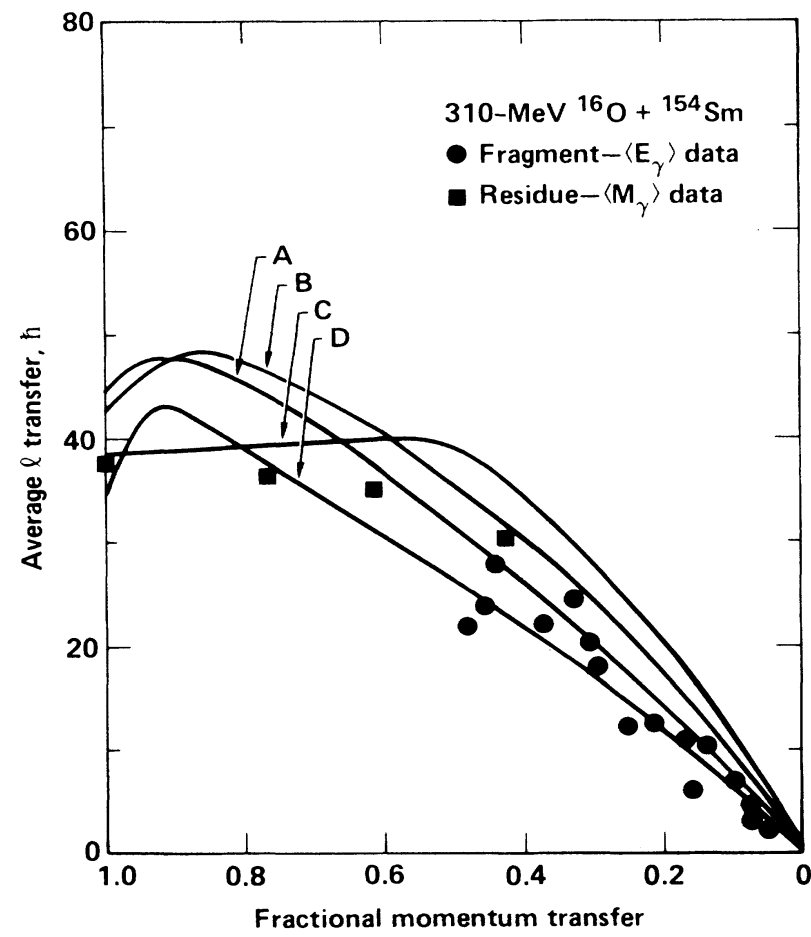

FIG. 13. Average transferred angular momentum as a function of fractional linear momentum transfer for the reactions of $310 \mathrm{MeV}{ }^{16} \mathrm{O}$ with ${ }^{154} \mathrm{Sm}$. The results have been corrected for missing momentum-see text. Theoretical predictions of several models are also presented. Curves: $A$, Wilczynski sum-rule mode; $B$, Harvey-Murphy overlap mode; $C$, Harvey's nucleonnucleon collision model with a nuclear mean free path of $13 \mathrm{fm}$; and $D$, Cole's nucleon-nucleon collision model. the fractional momentum transfer. This formula assumes that the missing mass is ejected straight forward with the beam velocity.

While the asymmetry of the residue velocity distribution clearly indicates a range of momentum transfers, the fluctuations resulting from particle evaporation broaden the primary distribution significantly. As a result, the actual range of momentum transfers represented by the data may be somewhat narrower. Note also that the two experimental methods complement each other very well. For the lowest momentum transfers the experimental difficulties of detecting the corresponding heavier residues favor the ejectile observation. In contrast, for the highest momentum transfers the ejectile method is less useful since the small primary ejectiles may be difficult to discern from sequential breakup products of larger ones.

For comparison with the data, Fig. 13 contains the results of several different model calculations. The sumrule and geometric overlap model predictions presented in Fig. 12 appear once again in this figure recast in terms of momentum transfer. As observed previously, they lead to similar predictions even though the initial partial waves involved are very different. This is a direct consequence of the different assumptions regarding the fraction of angular momentum carried by the captured particles. The derivation of the initial partial waves from the data will depend very sensitively on these assumptions. Results of the microscopic model calculations of the angular momentum transfer as a function of linear momentum transfer for our system by Harvey ${ }^{24}$ and $\mathrm{Cole}^{13}$ are also presented in Fig. 13.

Within the framework of the microscopic model of Harvey, the calculation shown in Fig. 13 corresponds to a choice of a mean free path ( $\mathrm{mfp}$ ) for nucleons in the nucleus of $13 \mathrm{fm}$. In the Monte Carlo calculations of fragment cross sections in Ref. 12, a nucleon $\mathrm{mfp}$ of $5 \mathrm{fm}$ was assumed. However, the cross sections for projectile-like fragments are quite insensitive to the value of the $\mathrm{mfp}$ and do not preclude values substantially different from $5 \mathrm{fm}$. Figure 14 shows that the calculated $l$-transfer at large momentum transfer are quite sensitive to the assumed nucleon mfp. Best agreement with the present results is obtained with a mfp of about $13 \mathrm{fm}$. The calculated values of the complete fusion cross section are also sensitive to the $\mathrm{mfp}$. The choice of $13 \mathrm{fm}$ predicts about $600 \mathrm{mb}$ for complete and near-complete fusion which is in reasonable agreement with the systematics. A value of $6.5 \mathrm{fm}$ gives a calculated cross section that is about twice as large. It should also be pointed out that a nucleon $\mathrm{mfp}$ of $13 \mathrm{fm}$ in the nuclear interior is consistent with the systematics of the volume part of the imaginary optical potential, ${ }^{24}$ and with a recent calculation by Blin et al. ${ }^{31}$

The Monte Carlo calculation with a $\mathrm{mfp}$ of $13 \mathrm{fm}$ reflects the relatively constant experimental values of the average $l$ transfer at high momentum transfer. As we have already indicated, the broadening of the primary residue velocity distribution by evaporation may lead to ascribing the observed $\gamma$ multiplicities corresponding to central collisions with the same linear momentum transfer, to a range of momentum transfers. This could create an artificial flattening of the curve of $\left\langle l_{t}\right\rangle$ versus 


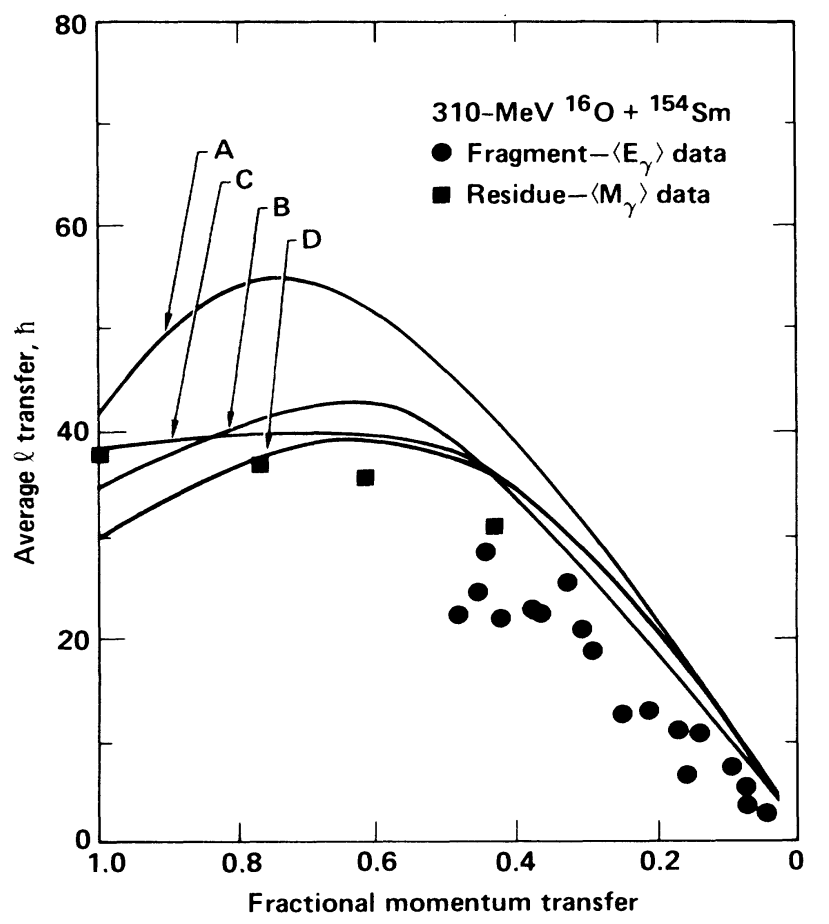

FIG. 14. Same experimental results as in Fig. 13. The curves show predictions of Harvey's microscopic model with different values of the nucleon mean free path. Curves $A-D$ correspond to mean free path values of $6.5,8,13$, and $14 \mathrm{fm}$, respectively.

fractional momentum transfer at high momentum transfers. It should also be noted that the region just below total momentum transfer may also have significant contributions from processes such as PEP (Ref. 32) and inertial emission (Ref. 33) which are expected to involve initial $l$-wave distributions extending considerably below the critical angular momentum for fusion. The fractional momentum processes of the "massive transfer" type described by the models discussed so far involve angular momenta above the fusion limit. In addition, the onset of fission deexcitation of the nuclei having the largest angular momenta, $\geq 60 \hbar$, may act to restrict the population of composite nuclei leading to heavy residues. Neither the sum-rule model nor the geometric overlap model predicts a large population of such fissioning nuclei in incomplete fusion reactions.

Finally, as an alternative way of viewing the data of Fig. 13, we note that if the actual relationship between the fractional mass transfer and fractional angular momentum transfer were understood, the data could be recast in terms of the initial partial waves or impact parameter contributing to particular mass or momentum transfers.

It is useful in this context to derive a simple approximation to the geometry of the transfer reaction. We may write, assuming spinless particles,

$$
l_{i}=l_{t}+l_{e},
$$

where

$$
\begin{aligned}
& l_{t}=\mu_{t} v_{\mathrm{vel}} r_{t}, \\
& l_{e}=\mu_{e} v_{\mathrm{rel}} r_{e} .
\end{aligned}
$$

Here, $\mu_{t}$ and $\mu_{e}$ are the reduced mass corresponding to the transferred mass $m_{t}$ and ejectile mass $m_{e}$, respectively, $r_{t}$ and $r_{e}$ are their relative distances from the center of the target, and $v_{\text {rel }}$ is the relative velocity at contact.

Assuming the vectors to be collinear, we may write

$$
l_{i}=l_{t}+\mu_{e} v_{\mathrm{rel}} r_{e},
$$

or

$$
l_{i}=l_{t}\left(1+\frac{r_{e} \mu_{e}}{r_{t} \mu_{t}}\right) .
$$

The same formula is presented by Bantel. ${ }^{34}$ Since we deal with the correlation between linear momentum transfer and angular momentum transfer, we note here that if it is assumed that mass transfer and linear momentum transfer are directly correlated, we may write

$$
\frac{\mu_{e}}{\mu_{t}} \simeq \frac{m_{e}}{m_{t}}=\frac{P_{i}-P_{t}}{P_{t}},
$$

where $P_{i}$ is the initial projectile momentum and $P_{t}$ is the transferred linear momentum. Then,

$$
l_{i}=l_{t}\left[1+\frac{r_{e}\left(P_{i}-P_{t}\right)}{r_{t} P_{t}}\right] \text {. }
$$

Equation (9) provides a simple first order approach to deriving the initial partial wave from the observed angular momentum transfer. Using the geometric overlap model, one can estimate that $r_{e} / r_{t}$ is about 1.4 for a wide range of mass transfers in the reactions of ${ }^{16} \mathrm{O}$ with ${ }^{154} \mathrm{Sm}$, while if $r_{e} / r_{t}$ is set equal to 1 , the assumption of the sum-rule model is reproduced.

It would be possible then, using such simple assumptions, to derive from the data of Fig. 13 a first order reconstruction of the average initial partial wave associated with each fractional momentum transfer. From these data alone, however, it does not appear possible, a priori, to distinguish between the two different prescriptions for this reconstruction. Nevertheless, if we consider that models of the overlap type may be more appropriate in the intermediate energy region, as discussed below, then the approximate reconstruction from the data given above would imply initial partial waves some $20 \%$ lower in magnitude than those calculated by the Monte Carlo nucleon-nucleon collision model of Harvey or by the overlap version of Ref. 11 [see Fig. 10(b)]. The detailed Monte Carlo calculations starting from the higher partial waves and using a mfp of $13 \mathrm{fm}$, however, do lead to $l$-transfer predictions in reasonable agreement with experiment, as shown in Fig. 13.

\section{SUMMARY AND CONCLUSION}

Using two different experimental techniques, we have established a correlation between linear momentum transfer and angular momentum transfer in the reactions of ${ }^{16} \mathrm{O}$ with ${ }^{154} \mathrm{Sm}$. The results have been compared with 
several models which predict the fractional transfers of these two quantities to result from incomplete fusion reactions. Models ascribing these reactions to very different initial partial waves predict rather similar values for the transferred angular momentum, reflecting primarily the very different assumptions regarding the correlation between the fractional mass transfer and fractional angular momentum transfer. At intermediate energies, where the reactions take place on a timescale comparable to the transit times of nucleons in a nucleus, the association of the transferred nucleons with the contact region between the two interacting nuclei appears quite reasonable. One would expect then that the fractional angular momentum transfer is less than the fractional mass transfer since the escaping cluster has an effectively higher interaction radius than the captured mass. Given these considerations and the success of the geometric overlap model in explaining isotope yields and excitation functions for primary ejectiles in a similar system, models of the overlap type may be most appropriate in this energy range. This does not rule out the possibility that intrinsic properties of a cluster might also come into play. However, if the captured mass is viewed as behaving substantially as individual nucleons in the spirit of the models of Refs. 12 and 13, the application of such mean field limits would not appear appropriate.

\section{ACKNOWLEDGMENTS}

Very fruitful conversations with J. Cole and B. G. Harvey contributed greatly to this work. This research was supported by the U.S. Department of Energy and the Robert A. Welch Foundation. Work by M. N. Namboodiri was performed in part at Lawrence Livermore $\mathrm{Na}$ tional Laboratory under the auspices of the U.S. Department of Energy under Contract No. W-7405-ENG-48.
*Present address: Lawrence Livermore National Laboratory, Livermore, CA 94550.

†Present address: Bhabha Atomic Research Centre, Trombay, Bombay, India.

†Present address: Washington University, St. Louis, MO 63130.

\$Present address: University of Notre Dame, Notre Dame, IN 46556.

** Present address: Hope College, Holland, MI 49423.

${ }^{\dagger \dagger}$ Present address: University of Washington, Seattle, WA 98195.

${ }^{1}$ V. E. Viola, B. B. Back, K. L. Wolf, T. C. Awes, C. K. Gelbke, and H. Breuer, Phys. Rev. C 26, 178 (1982).

${ }^{2}$ H. Morgenstern, W. Bohne, K. Grabisch, D. G. Kovar, and H. Lehr, Phys. Lett. 113B, 463 (1983); H. Moregenstern, W. Bohne, K. Grabisch, H. Lehr, and W. Stoffler, Z. Phys. 313, 39 (1983).

${ }^{3}$ R. Stokstad, Y. Chan, M. Murphy, I. Tserruya, S. Wald, and A. Budzanowski, Lawrence Berkeley Laboratory Report No. LBL-15874, 1983.

${ }^{4}$ K. A. Geoffroy, D. G. Sarantites, M. L. Halbert, D. C. Hensley, R. A. Dayras, and J. H. Barker, Phys. Rev. Lett. 43, 1303 (1979).

5J. R. Huizenga, J. R. Birkelund, L. E. Tubbs, D. Hilscher, U. Jahnke, H. Rossner, B. Gebauer, and H. Lettau, Phys. Rev. C 28, 1853 (1983); L. E. Tubbs, J. R. Birkelund, J. R. Huizenga, D. Hilscher, U. Jahnke, H. Rossner, and B. Gebauer, University of Rochester Report No. UR-NSRL-288, 1984 (unpublished).

${ }^{6}$ C. C. Hsu, D. J. Morissey, L. W. Richardson, G. J. Wozniak, and L. G. Moretto, Lawrence Berkeley Laboratory Report No. LBL-12519, 1980.

${ }^{7}$ A. Gavron, J. Boissevain, H. C. Britt, K. Eskola, P. Eskola, M. M. Fowler, H. Ohm, J. B. Wilhelmy, T. C. Awes, R. L. Ferguson, F. E. Obenshain, F. Plasil, G. R. Young, and S. Wald, Phys. Rev. C 30, 1550 (1984).

${ }^{8}$ K. Siwek-Wilczynska, E. H. deMarchie van Voorthusyen, J. van Popta, R. H. Siemssen, and J. Wilczynski, Phys. Rev. Lett. 42, 1599 (1979).

${ }^{9}$ J. Wilczynski, K. Siwek-Wilczynska, J. Van Driel, S. Gonggrijp, C. J. M. Hageman, R. V. F. Janssens, J. Lukasiak, R. H. Siemssen, and S. Y. Van der Werf, Nucl. Phys. A373, 109 (1982).
${ }^{10}$ B. G. Harvery and M. J. Murphy, Phys. Lett. 130B, 373 (1983).

${ }^{11}$ B. G. Harvey and H. Homeyer, Lawrence Berkeley Laboratory Report No. LBL-16882, 1984 (unpublished).

${ }^{12}$ B. G. Harvey, Nucl. Phys. A444, 498 (1985).

${ }^{13}$ A. J. Cole, Z. Phys. A 322, 315 (1985), and private communication.

${ }^{14}$ T. Udagawa and T. Tamura, Phys. Rev. Lett. 45, 1311 (1980).

${ }^{15} \mathrm{X}-\mathrm{H}$. Li, T. Udagawa, and T. Tamura, Phys. Rev. C 30, 1895 (1984).

${ }^{16}$ A. K. Kerman and K. McVoy, Ann. Phys. (N.Y.) 122, 19 (1979).

${ }^{17}$ G. W. Butler, A. M. Poskanzer, and D. W. Landis, Nucl. Instrum. Methods 89, 189 (1970).

${ }^{18}$ M. N. Namboodiri, J. B. Natowitz, P. Kasiraj, R. Eggers, L. Adler, P. Gonthier, C. Cerruti, and S. Simon, Phys. Rev. C 20, 982 (1979).

${ }^{19}$ J. P. Sullivan, U. Garg, D. R. Haenni, Y.-W. Lui, Y. Toba, K. Yates, and D. H. Youngblood, Cyclotron Institute, Texas A\&M University Annual report, 1984-85, p. 103 (unpublished).

${ }^{20}$ R. P. Schmitt, G. Mouchaty, and D. R. Haenni, unpublished results.

${ }^{21}$ R. P. Schmitt, G. Mouchaty, and D. R. Haenni, Nucl. Phys. A427, 614 (1984).

${ }^{22}$ D. J. Morissey, W. Benenson, E. Kashy, C. Bloch, M. Lowe, R. A. Blue, R. M. Ronningen, B. Sherril, U. Utsonomiya, and I. Kelsen, Phys. Rev. C 32, 817 (1985).

${ }^{23}$ J. Gomez del Campo and R. G. Stokstead, Oak Ridge National Laboratory Report No. TM-7295, 1981.

${ }^{24}$ B. G. Harvey, private communication.

${ }^{25}$ T. C. Awes, S. Saini, G. Poggi, C. K. Gelbke, D. Cha, R. Legrain, and G. D. Westfall, Phys. Rev. C 25, 2361 (1982).

${ }^{26}$ H. Delegrange, D. Logan, M. F. Rivet, M. Rajagopalan, J. M. Alexander, M. S. Zisman, M. Kaplan, and J. W. Ball, Phys. Rev. Lett. 43, 1490 (1979).

${ }^{27}$ P. L. Gonthier, H. Ho, M. N. Namboodiri, J. B. Natowitz, L. Adler, S. Simon, K. Hagel, S. Kniffen, and A. Khodai, Nucl. Phys. A411, 289 (1983).

${ }^{28}$ B. B. Back, K. L. Wolf, A. C. Mignerey, C. K. Gelbke, T. C. Awes, H. Breuer, V. E. Viola, and P. Dyer, Phys. Rev. C 22, 1927 (1980). 
${ }^{29}$ C. K. Gelbke, C. Olmer, M. Buenerd, D. L. Hendrie, J. Mahoney, M. C. Mermaz, and D. K. Scott, Phys. Rep. 42, 312 (1978).

${ }^{30}$ B. Borderie, M. F. Rivet, C. Cabot, D. Fabris, D. Gardes, H. Gauvin, F. Hanappe, and J. Peter, Z. Phys. 316, 243 (1984).

${ }^{31}$ A. H. Blin, R. W. Hasse, B. Hiller, and P. Schuck, Phys. Lett.
161B, 211 (1985).

32J. P. Bondorf, J. N. De, G. Fai, A. O. T. Karvinen, B. Jakobsson, and J. Randrup, Nucl. Phys. A333, 285 (1980).

${ }^{33}$ H. Tricoire, Z. Phys. A 317, 347 (1984).

${ }^{34}$ M. Bantel, Ph.D. thesis, Heidelberg University, 1985 (unpublished). 